\title{
A Study of Single Electron Transistor (SET)
}

\author{
Monika Gupta \\ New Horizon College of Engineering, Bangalore, India
}

\begin{abstract}
Recent research in SET gives new ideas which are going to revolutionize the random access memory and digital data storage technologies. The goal of this paper is to discuss about the basic physics, applications of nano electronic device 'Single electron transistor [SET]' which is capable of controlling the transport of only one electron. Single-electron transistor (SET) is a key element of current research area of nanotechnology which can offer low power consumption and high operating speed. The single electron transistor is a new type of switching device that uses controlled electron tunneling to amplify current. and retains its scalability even on an atomic scale. Here, scalability means that the performance of electronic devices increases with a decrease of the device dimensions. In this paper, we also focus on some basic device characteristics like 'Coulomb blockade', single electron tunneling effect \& 'Coulomb staircase' on which this Single electron transistor [SET] works and the basic comparison of SET \& MOSFET characteristics and also its [SET] advantages as well as disadvantages to make a clear picture about the reason behind its popularity in the field of nanoelectronics.
\end{abstract}

Keywords: Single-electron tunneling, Coulomb blockade, Coulomb staircase, Single electron Transistor, Quantum dot, Nanoelectronics.

\section{Introduction}

In today's digital integrated circuit architectures, transistors serve as circuit switches to charge and discharge capacitors to the required logic voltage levels[1]. A transistor is a three terminal semiconductor device used to amplify and switch electronic signals and electrical power. It has been observed that the Scaling down of electronic device sizes has been the fundamental strategy for improving the performance of ultra-large-scale integrated circuits (ULSIs). Metal-oxidesemiconductor field-effect transistors (MOSFETs) have been the most prevalent electron devices for ULSI applications, and thus the scaling down of the sizes of MOSFETs [1][2] has been the basis of the development of the semiconductor industry for the last 30 years. However, in the early years of the 21 st century, the scaling of CMOSFETs is entering the deep sub-50 $\mathrm{nm}$ regime [3]. In this deep-nanoscaled regime, fundamental limits of CMOSFETs and technological challenges with regard to the scaling of CMOSFETs are encountered [4]. On the other hand, quantum-mechanical effects are expected to be effective in these small structured devices. Therefore, in order to extend the prodigious progress of LSI performance, it is essential to introduce a new device having an operation principle that is effective in smaller dimensions and which may utilize the quantummechanical effects, and thus provide a new functionality beyond that attainable with CMOSFETs.

Single-electron devices [5] are promising as new nanoscaled devices because single-electron devices retain their scalability even on an atomic scale and moreover, they can control the motion of even a single electron. Therefore, if the single-electron devices are used as ULSI elements, the ULSI will have the attributes of extremely high integration and extremely low power consumption. Power consumption is roughly proportional to the electron number transferred from voltage source to the ground in logic operations. Therefore, the utilization of single electron devices in ULSIs is expected to reduce the power consumption of ULSIs. SET is said to be the tiny transistor with tiniest power consumption. Since the technology reaches nano size, the behavior of a nanoelectronic single electron transistor (SET) is controlled by the quantum mechanical effects.
The rest of this paper is organized as follows: Section 2 states about the SET schematic and working. Section 3 gives the description of principle of Single electron tunneling \& Coulomb Blockade. Section 4 presents the I-V characteristics of single electron transistors. Section 5 presents the comparison between SET and MOSFET. Section 6 presents the comparison between SET and CMOS. Section 7 presents the applications of Single Electron Transistor. Finally, Section $8 \& 9$ describes the advantages \& disadvantages of SET followed by conclusion.

\section{SET Schematic and its Working}

Single-electron transistors (SETs) are essential elements of ULSI logic circuit design. A three terminal switching device in which the effect of coulomb blockade can be observed is generally known as single-electron transistor. Unlike field effect transistors, single-electron transistors are based on an intrinsically quantum phenomenon which is known as „Tunnel effect.'

This tunnel effect may be observed when two metallic electrodes are separated by an insulating barrier known as tunnel junction. These three terminal switching devices can transfer electrons form source to drain one by one. The schematic structure of SETs is shown in Fig. 1. 


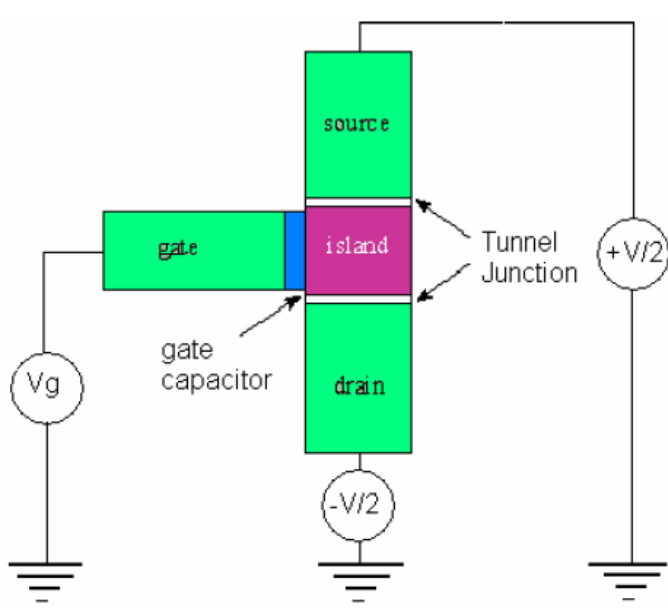

Figure 1: Schematic structure of a SET

Quantum dot which is less than $100 \mathrm{~nm}$ in diameter is a mesoscopic system in which the electrostatic energy or coulomb energy can be changed due to removal or addition of a single electron that is greater than the thermal energy and can control the electron transport into and out of the quantum dot. In other words, Quantum dot is a small conducting island that contains a tunable number of electrons occupying discrete orbitals.

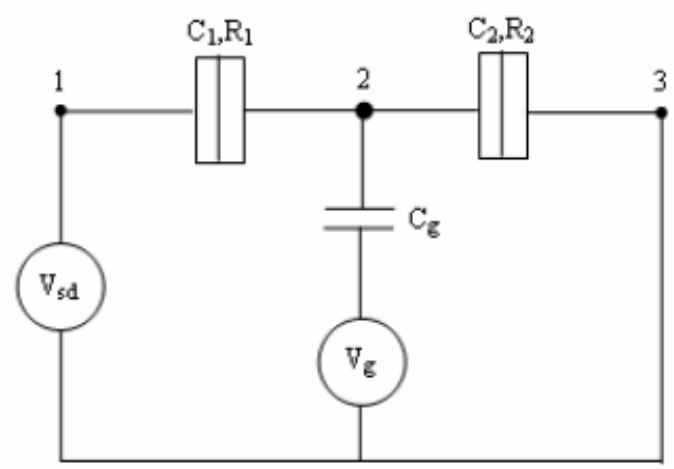

Figure 2: Single electron transistor circuits with Single Island

As shown in Fig. 2 \& 3, Single electron transistor circuits are made of small tunnel junctions, capacitances, and voltage sources. A gate voltage $\mathrm{V}_{\mathrm{g}}$ is used to control the opening and closing of the SET or in other words, it controls one-by-one electron transfer. Here, a tunneling electron can be described as a discrete charge due to stochastic nature of a tunneling event. In Figures $2 \& 3$, node 1 represents source electrode, node 2 (and 4) is island, and node 3 represents drain electrode. The tunnel junctions are located in between these nodes, which are described by tunnel capacitance and tunnel resistance. The single electron tunneling theory depends on the ,Orthodox theory ${ }^{\text {ee }}$ which focuses on charging of the small conducting island of diameter $\leq 100 \mathrm{~nm}$. The capacitance of island is given by the equation for a Conducting spherical capacitance

$$
\mathrm{C}=2 \pi \epsilon_{0} \mathrm{Cd}
$$

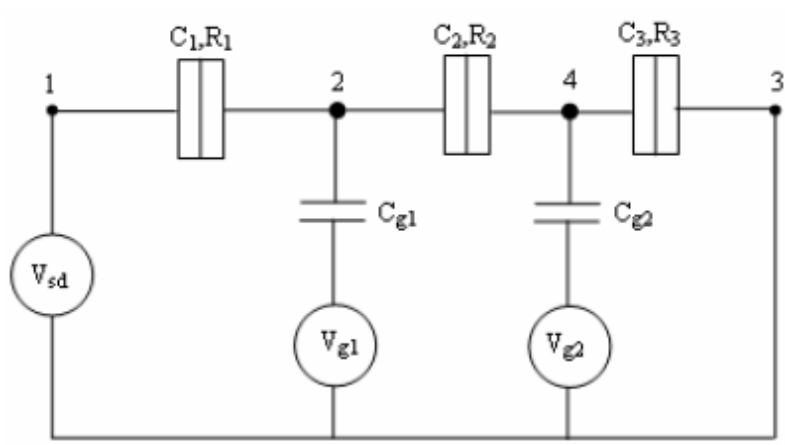

Figure 3: Single electron transistor circuits with Dual Island as SET is composed of two tunnel junctions along with one island which are capatively coupled to gate electrode. SET can electrostatically be controlled by the gate capacitance.

Thus the total capacitance of the island is given by

$$
\mathrm{C}_{\Sigma}=\mathrm{C}_{1}+\mathrm{C}_{2}+\mathrm{C}_{\mathrm{g}}
$$

Thus, such a set up is called single electron transistor.

\section{Principle of Single-Electron Tunneling \& Coulomb Blockade}

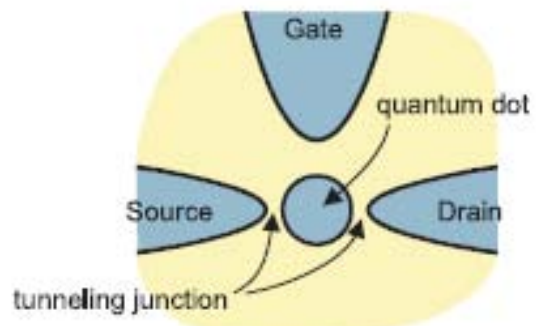

Figure 4: Schematic structure of SET showing tunnel junctions

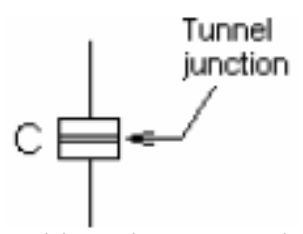

Figure 5: Tunnel junction or Insulating Barrier

As shown in Fig $4 \& 5$, a tunnel junction is considered as a thin insulating barrier between two conducting electrodes. In case of classical electrodynamics, no current can flow through an insulating barrier. But according to the quantum mechanics approach, there is some probability (i.e. greater than zero) for an electron located at one side of the barrier (tunnel junction) to reach the other side. Thus, the transfer of electrons through the barriers between the quantum dots would result in charging of the neighboring quantum dots. Now, this would result in an increase of the electrostatic energy which is given by

$$
\mathrm{E}_{\mathrm{c}}=\frac{\mathrm{e}^{2}}{2 \mathrm{c}}
$$

Where $\mathrm{C}$ is the effective capacitance of the island. Later on, this electrostatic energy became known as Coulomb charging energy or coulomb blockade energy. This coulomb blockade 


\section{International Journal of Science and Research (IJSR) \\ ISSN (Online): 2319-7064}

Index Copernicus Value (2013): 6.14 | Impact Factor (2014): 5.611

energy is the repelling energy of previous electron present in the island to the next electron coming towards the island. In case of a tiny system, the capacitance $\mathrm{C}$ of the island is very small. Thus, according to equation (3), the coulomb charging or blockade energy $\left(E_{c}\right)$ will be very high and due to this reason, electrons are unable to move simultaneously, but pass one-by-one. Thus, this coulomb charging energy or coulomb blockade energy is responsible for the suppression of the electrons transfer simultaneously. This phenomenon is known as "Coulomb blockade." The suppression of electron transfer can be removed by one of these two possible cases:

(a) When the coulomb charging energy is overcome by thermal excitations at a temperature T, i.e

$$
\mathrm{T} \sim \mathrm{T}_{0}=\frac{\mathrm{E}_{\mathrm{c}}}{\mathrm{K}_{\mathrm{g}}}
$$

(b) When the coulomb charging energy is overcome by an externally applied voltage $\mathrm{V}$, i.e.

$$
V \sim V_{t}=\frac{E_{c}}{e}=\frac{e}{2 C}
$$

Where, is known as „Threshold voltage ${ }^{\text {ee }}$ which is defined as an applied voltage that is just sufficient to increase the energy of electron above the coulomb blockade of tunneling so that the current can start to flow though the tunnel junction (Barrier) . This suppression of electron transfer is normally termed as ,Coulomb blockade (CB) of tunnelingee. Thus, it is clear that if the voltage $\mathrm{V}$ is less than the threshold voltage $V_{t}$, the system is in Coulomb blockade (CB) state. In case, when the voltage $\mathrm{V}$ exceeds $\mathrm{V}_{\mathrm{t}}$, an electron tunnels through the barrier (tunnel junction) into the quantum dot. Fig. $2 \& 3$ represents the single electron circuits with Single Island and with Double Island. For making the concept of coulomb blockade of tunneling more clearly, we again consider the single electron circuits in which on applying bias voltage, there will be a current flow. Now, if we avoid additional effects, in that case, the first order approximation tunneling current is proportional to the applied bias voltage. In other words, we can say that a tunnel junction behaves like a resistor of a constant value which depends on the barrier thickness. Since, the tunnel junction is composed of two conductors and an insulating layer in between these two conductors. Therefore, tunnel junction is described by the tunnel resistance $\mathrm{R}$ and tunnel capacitance $\mathrm{C}$. In this case, tunnel junction acts as a capacitor and the insulating layer works as a dielectric medium for tunnel capacitor $\mathrm{C}$. The current flowing through a tunnel junction is a series of events in which only one electron passes through the tunnel junction because of discrete nature of charge. As electron tunnels the junction, tunnel capacitance is charged with an elementary charge building up a voltage which is represented by $\mathrm{V}=$ $\mathrm{e} / \mathrm{C}$. If the capacitance of the tunnel junction is very small, the voltage developed in the tunnel junction may be sufficient to prevent another electron to tunnel. Now, in this situation, the electric current is suppressed if the bias voltage is lower than the voltage developed in the tunnel junction. Thus the increment of the tunnel junction resistance around zero bias is considered as the coulomb blockade. Therefore, coulomb blockade may be defined as the increased tunnel junction resistance at very low bias voltages of an electronic device which consists of at least one low capacitance tunnel junction.
The coulomb blockade can be achieved only if, in case, when the following three conditions meet:

1. The bias voltage must be lower than the elementary charge divided by the self-capacitance of the island. i.e.

$$
\mathrm{V}_{\text {bias }}<\mathrm{e} / \mathrm{C}
$$

2. The thermal energy $K_{B} T$ must be below the charging energy i.e.

$\mathrm{K}_{\mathrm{B}} \mathrm{T}<\mathrm{e}^{2} / \mathrm{C}$; or else the electron will be able to pass the quantum dot (QD) via thermal excitation.

3. The Tunneling resistance $\left(\mathrm{R}_{\mathrm{T}}\right)$ should be greater than $\mathrm{h} / 2 \pi \mathrm{e}^{2}$, which is derived from ,Heisenberg's uncertainty principle ${ }^{e e}$. i.e.

$$
\mathrm{R}_{\mathrm{T}}>\mathrm{h} / 2 \pi \mathrm{e}^{2}=25813 \Omega .
$$

This is the required condition for tunnel resistance.

\section{I-V Characteristics of SET}

Fig. 6(a) shows the I-V Characteristics for the symmetric junction circuit of single electron transistor where $\mathrm{C}_{1}=\mathrm{C}_{2}$ and $\mathrm{R}_{1}=\mathrm{R}_{2}$. It is clear from the I-V characteristics of the SET that for $|\mathrm{V}|<\mathrm{e} / \mathrm{C}_{\Sigma}$, the current is zero. This state is called Coulomb blockade that suppresses the tunneling of single electron in case of low bias condition. Now, if the externally applied junction voltage $\mathrm{V}$ is increased up to a level that is above the threshold voltage by charging energy, this effect of Coulomb blockade can be removed and the current flows. In this situation, the junction behaves like a resistor. The sequential entrance and leaving of an electron from one junction to another is generally known as „Correlated tunneling of electrons ${ }^{\text {ee }}$.

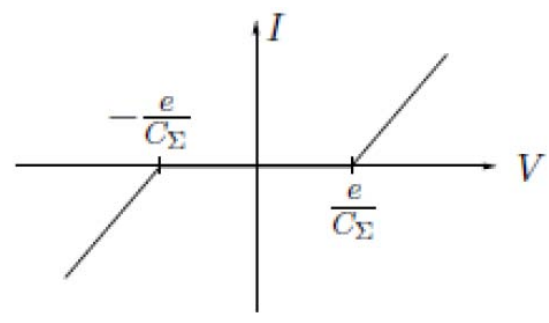

Figure 6(a): I-Vcharacteristics of SET for symmetric junction.

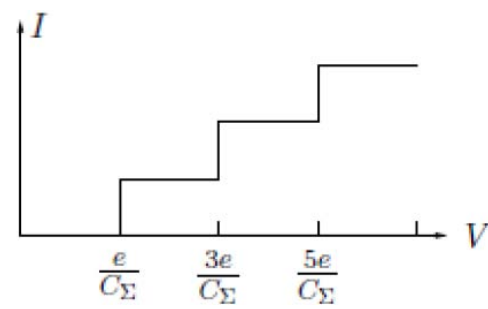

Figure 6(b): I-V Characteristics of the SET for asymmetric junction representing ,coulomb Staircase ${ }^{\text {ec }}$ state.

Fig. 6(b) represent the I-V Characteristics for a highly asymmetric junction circuit for $\mathrm{R}_{1}<<\mathrm{R}_{2}$. In this case, the charge carriers i.e. electrons enter through one junction and then escape to second junction due to the presence of high resistance. Now, electrons moves from one junction to 


\section{International Journal of Science and Research (IJSR) \\ ISSN (Online): 2319-7064 \\ Index Copernicus Value (2013): 6.14 | Impact Factor (2014): 5.611}

another very rapidly. Thus this rapid movement of excess electrons from one junction to another raises the total charge of the island. If the bias is increased, it will tend to increase the population of electrons in the island. In this case the I-V Curve represents Stair-like characteristics, which are commonly referred to as the „Coulomb Staircase ${ }^{e e}$.[1]

\section{Applications of SET}

\subsection{Charge Sensor}

The Single-electron transistors (SETs) are efficient charge sensors for reading out spin. To investigate their capacitive parameters, which are related to the signal-to-noise ratio (SNR) during quantum bits readout, twin silicon single (quantum dots) QDs were fabricated using a lithographic process on a silicon-on insulator substrate. Since the configuration and dimensions of the QDs could be determined by direct imaging, the theoretical capacitive parameters could be compared to the measured values. Good agreement was found between the calculated and measured value, which confirms the validity of the calculation method. The results indicated that decreasing the SET diameter reduces the capacitive coupling between quantum bits but increases the signal-to-noise ratio for both $\mathrm{dc}$ and radio frequency single shot measurements. Since these results are independent of the device materials, they are useful for establishing guidelines for the design of SET charge sensors in lateral QD-SET structures based on a two dimensional electron gas.

\subsection{Detection Of Infrared Radiation}

The single-electron transistor can also be used to detect infrared signals at room temperature. Generally, the response differs from that the well-known Tien-Gordon theory of photon-assisted tunneling. In fact, this is based on the assumption of uncorrelated tunneling events, while in singleelectron systems the electron transfer is typically correlated [6].

By exciting electrons over an electrically induced energy barrier, both the range of detectable wavelengths and the sensitivity of the device can be controlled. The sensor works when an infrared signal excites conduction band electrons in a $25 \mathrm{~nm}$ deep electron reservoir. A silicon insulator channel measuring $40 \times 400 \mathrm{~nm}$ is placed next to the reservoir to increase the number of excited electrons. A poly-silicon lower gate then turns off the transistor and electrically forms an energy barrier, creating a storage node on the other side. Electrons with energy greater than the height of the barrier are injected into the storage node, where they are read as changes in current flowing through the transistor.

\subsection{Ultrasensitive Microwave Detector}

Single Electron Transistor can work an Ultrasensitive Microwave Detector island is weakly coupled to a bias circuit through two small capacitance tunnel junctions and a capacitive gate. At low bias voltages and temperatures, a single quasi-particle may only be introduced to the island through photon-assisted tunneling. Once this occurs, the quasi particle is trapped on the island because it takes a relatively long time for this specific quasi particle to tunnel off. While it is trapped, charge is transported through the system two electrons at a time. Since the photon-assisted transition merely switches the detector current on this device is not limited to one electron tunneled through the system per absorbed photon. This makes the device an extremely sensitive and potentially useful detector of microwave radiation.

\subsection{Supersensitive Electrometer}

The high sensitivity of single-electron transistors have enabled them as electrometers in unique physical experiments. For example, they have made possible unambiguous observations of the parity effects in superconductors. Absolute measurements of extremely low dc currents $\left(\sim 10^{-20}\right.$ A) have been demonstrated. The transistors have also been used in the first measurements of single-electron effects in single-electron boxes and traps. A modified version of the transistor has been used for the first proof of the existence of fractional-charge excitations in the fractional quantum hall effect.

\subsection{Single-Electron Spectroscopy}

One of the most important application of single-electron electrometry is the possibility of measuring the electron addition energies (and hence the energy level distribution) in quantum dots and other nanoscale objects.

\subsection{Temperature Standards}

Theoretical analysis based on the orthodox theory has shown that $\Delta \mathrm{V}=5.44 \mathrm{Nk}_{\mathrm{B}} \mathrm{T} / \mathrm{e}$ is surprisingly stable with respect to almost any variations of the array parameters (with the important exception of a substantial spread in the junctions resistances) providing a remarkable opportunity to use the arrays for absolute thermometry, since the fundamental constants are known with high accuracy. Each particular array may give high $(1 \%)$ accuracy of within less than one decade of temperature variations, but for arrays with different island size, these ranges may be shifted and overlap. Thus, it is possible to have an absolute standard of temperature with a very broad (say, two-decade) total range from several circuits fabricated on a single chip. This development is very encouraging, but since all this work is recent, some time is needed to see whether these new devices will be able to compete with (or even replace) the established temperature standards.

\subsection{Programmable Single Electron Transistor Logic}

An SET having non volatile memory function is a key for the programmable SET logic. The half period phase shift makes the function of SET complimentary to the conventional SETs. As a result SETs having non-volatile memory function have the functionality of both the conventional (n-MOS like) SETs and the complementary (pMOS like) SETs. By utilising this fact the function of SET circuit can be programmed on the basis of function stored by the memory function. The charged around the QD of the SET namely an SET island shift the phase of coulomb oscillation, the writing/erasing operation of memory function which inject/eject charge to/from the memory node near the SET Island, makes it possible to tune the phase of coulomb oscillation. If the injected charge is adequate the phase shift is half period of the coulomb oscillation. 


\section{Comparison between SET and MOSFET}

However, the structure of Single electron transistors [SETs] is almost same as that of MOSFETs but still there are some differences between the SETs and MOSFETs such as SETs have tunnel junction in place of $p-n$ junction of the MOSFETs and a small conducting island [quantum dot] in place of channel region of the MOSFETs.
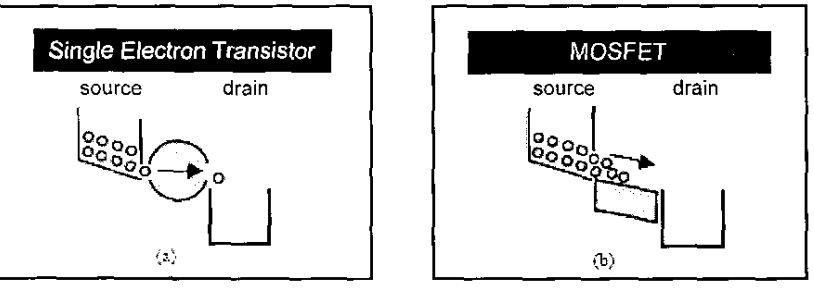

Figure 7: Electron tunneling through the channel (a) one-byone in SET (b) Transfer of many electrons simultaneously through the channel in MOSFET.

In SET the tunneling electrons are transferred one-by-one through the island from source to drain due to the effect of Coulomb blockade whereas in MOSFET the number of electrons is transferred through the channel at a time. Thus many electrons simultaneously participate to the drain current. Due to the Coulomb blockade effect, an electron approaching a small negative charged region experiences the electrostatic repulsion by the previous electron in that region. This regulates the number of electrons one-by-one in the channel and hence the drain current varies accordingly. In case of SETs, the drain current $\left(\mathrm{I}_{\mathrm{d}}\right)$ does not depend on the number of electrons transferring through the channel or on the Fermi velocity. The $\mathrm{I}_{\mathrm{d}}-\mathrm{V}$ characteristic of SET is periodic which shows a finite drain current only for the specific gate voltages where the energies for $\mathrm{N}$ and $\mathrm{N}+1$ electron in the channel are degenerated. In case of FETs, the drain current $\left(\mathrm{I}_{\mathrm{d}}\right)$ depends on the number of electrons passes through the channel. i.e. more electrons in the channel, larger the drain current.

The common phenomenon in both of these two devices is the electrostatic effect that rules on both of them (i.e. SETs \& MOSFETs). But in case of SETs, electrons are not free to move from source to drain due to the presence of tunnel junctions. Unlike ideal FETs, the source and drain of the SETs are separated from the channel by sufficiently large resistances which are acting as tunnel barriers.

\section{Comparison between SET and CMOS}

The most studies carried out on SET based logic circuits works upon the tunnel junction using it to implement the SET as a switch equivalent of the MOS transistor [2]. This has the advantage that existing CMOS transistor based designs can easily be ported to SET technology. The drawbacks includes that the current transport though an open transistor still amounts for a large number of individual electrons dripping through the tunnel island junction. This is hence a far slower process than the transport of just one single electron through the same and consequently approaches that mimic the CMOS design style do not use the SET technology to its full potential.

Table 1: The theoretical comparisons between SET and CMOS

\begin{tabular}{|c|c|c|}
\hline Parameters & $\begin{array}{c}\text { CMOS } \\
\text { Circuit }\end{array}$ & SET circuit \\
\hline Maximal switching speed & $10-$ Oct & $15-$ Oct \\
\hline Supply voltage range & $100 \mathrm{~m}$ & $100 \mu \mathrm{V}$ \\
\hline Current range & $\mathrm{nA}$ & Few electron \\
\hline RBC sensitivity & None & $\gamma$ \\
\hline Maximum voltage gain & High & High \\
\hline Maximum operation temperature & $>300^{\circ}$ & Difficult at 300K \\
\hline
\end{tabular}

\section{Advantages of SET}

Following are the advantages of Single electron transistors (SETs):

- Low energy consumption

- High sensitivity

- Compact size

- High operating speed

- Simplified circuit

- Feature of reproducibility

- Simple principle of operation

- Straight forward co-integration with traditional CMOS circuits.[6]

\section{Disadvantages of SET}

Following are the disadvantage of Single-electron transistors

- Integration of SETs in a large scale: To operate SETS at room temperature, large quantities of mono dispersed Nano particles less than $10 \mathrm{~nm}$ in diameter must be synthesized. But, it is very hard to fabricate large quantities of SETs by traditional optical lithography and semiconducting process.

- It is difficult to link SETs with the outside environment.

- Practically difficult to fabricate Single electron transistors (SETs ) [6].

\section{Conclusions}

Single electron devices have already proved their value as tools in scientific research. There are strong efforts around the world to make the artificial atoms in SETs smaller, in order to raise the temperature at which charge quantization can be observed. These involve self assembly techniques and novel lithographic and oxidation methods whereby artificial atoms can be made nearly as small as natural ones. This is, of course, driven by an interest in using SETs for practical applications. However, as SETs get smaller, all of their energy scales can be larger, so it is very likely that new phenomena will emerge.

Many in the semiconductor field think that the industry is fast approaching the physical limits of transistor miniaturization. The major problem in modern transistors is power leakage leading to the generation of excessive heat from billions of transistors in close proximity. Only one thing is certain: if the pace of miniaturization continues 


\section{International Journal of Science and Research (IJSR) \\ ISSN (Online): 2319-7064}

Index Copernicus Value (2013): 6.14 | Impact Factor (2014): 5.611

unabated, the quantum properties of electrons will become crucial in determining the design of electronic devices before the end of the next decade. It is also one of the key building blocks needed to make quantum computers which promise exponential increases in processing speed over todayes computers through their use of the ,spin", or magnetic orientation, of individual electrons to represent data in their calculations. SETs have shown promising applications in metrology, tera hertz radiation diction and imaging. Because of natural small dimension, SET is a potential solution for continue silicon scaling. In addition, low power dissipation can be achieved in circuits composed of single-electron devices. Metallic structures can be fabricated with vertical and lateral islands and tunnel barrier structures at temperature up to $77 \mathrm{~K}$. GaAs and silicon based SETs have been already demonstrated. However, fabrication is still the most difficult challenge of SET application. Electron beam lithography and scanning probe techniques offer the best prospects for the future. Besides, some more esoteric techniques based on atomic particle deposition and colloid chemistry may also provide some benefits [5].

The future of research on SETs looks very bright. It now seems that electronics based on individual molecules and single-electron effects will replace conventional circuits based on scaled-down versions of field-effect transistors.

\section{References}

[1] Vinay Pratap Singh, Arun Agrawal, Shyam Babu Singh, "Analytical Discussion of Single Electron Transistor (SET)," International Journal of Soft Computing and Engineering (IJSCE), ISSN: 2231-2307, Volume-2, Issue-3, July 2012

[2] Shagun Pal, S. R. P. Sinha, Rahul Verma, "Performance Evaluation of Single Electron Transistor with CMOS Technology",International Journal of Emerging Technology and Advanced Engineering, (ISSN 22502459, ISO 9001:2008 Certified Journal, Volume 3, Issue 12, December 2013)

[3] Shilpa Goyal, Anu Tonk, "A Review towards Single Electron Transistor (SET)", International Journal of Advanced Research in Computer and Communication Engineering Vol. 4, Issue 5, May 2015

[4] Varun Mittal, "Basic introduction to Single Electron Transistor," IJRASET, ISSN: 2321-9653 Vol. 1 Issue I, August 2013

[5] Radha Krishnan, "Single Electron Transistors", International Journal of Scientific \& Engineering Research, ISSN 2229-5518, Volume 5, Issue 9, Sep2014

[6] Om Kumar and Manjit Kaur, "Single electron Transistor: Applications \& Problems ", International Journal of VLSI Design and Communication Systems, (VLSICS) Vol.1, No.4, December 2010

[7] A. Venkataratnam and A. K. Goel, "Design and simulation of Logic Circuits with Hybrid Archietectures of Single Electron Transistors and Conventional Devices", U.S. Army Research Laboratories, 2006 IEEE.

\section{Author Profile}

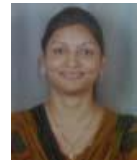

Monika Gupta received M.Tech Degree in VLSI Design (Gold Medalist) from MNIT Jaipur, PGDBM in Finance from Symbiosis Pune and B.E honors in E\&C Engineering with total rich work experience of $7.5 \mathrm{yrs}$ as an Academician. Currently working as an Assistant Prof. (Dept. of ECE) in New Horizon College of Engineering, Bangalore. 